\title{
Lie symmetries for two-dimensional charged particle motion
}

\author{
F. Haas and J. Goedert \\ Centro de Ciências Exatas e Tecnológicas, UNISINOS \\ Av. Unisinos, 950 \\ 93022-000 São Leopoldo, RS - Brazil
}

\begin{abstract}
We find the Lie point symmetries for non-relativistic two-dimensional charged particle motion. These symmetries comprise a quasi-invariance transformation, a time-dependent rotation, a time-dependent spatial translation and a dilatation. The associated electromagnetic fields satisfy a system of first-order linear partial differential equations. This system is solved exactly, yielding four classes of electromagnetic fields compatible with Lie point symmetries.
\end{abstract}

\section{Introduction}

Lorentz equations for non-relativistic charged particle motion constitute a very basic dynamical system whose symmetry structure deserves a detailed investigation. In a previous paper [1], we have studied the Noether point symmetries for two-dimensional non-relativistic charged particle motion. Here we make a systematic search of the Lie point symmetries associated with twodimensional non-relativistic charged particle motion under general electromagnetic fields. The reasons for this are two-fold. First, as is well known [2], the Lie point symmetry group is more general and contains the Noether 
point symmetry group of any given problem. In fact, the Lie point symmetries extends the class of Noether point symmetries of planar non-relativistic charged particle motion by the incorporation of an extra scale symmetry. Second, even if the Lie point symmetry approach does not give first integrals as directly as Noether's theorem, it does opens the possibility for reducing the number of relevant variables in the system. Reduction of variables is of importance in reducing the amount of computational time in numerical simulations. For example, in the numerical treatment of the Vlasov-Maxwell system in colisionless plasma physics [3, 4], it is highly desirable to know the most general electromagnetic field configurations having Lie point symmetry.

Herein, we consider planar non-relativistic charged particle motion under initially general electromagnetic field. The corresponding Lorentz equations read

$$
\begin{aligned}
& \ddot{x}=E_{1}(x, y, t)+\dot{y} B(x, y, t), \\
& \ddot{y}=E_{2}(x, y, t)-\dot{x} B(x, y, t),
\end{aligned}
$$

where $\mathbf{E}=\left(E_{1}(x, y, t), E_{2}(x, y, t), 0\right)$ is the planar electric field and $\mathbf{B}=$ $(0,0, B(x, y, t))$ is the perpendicular magnetic field. Unlike the usual approach to Lie symmetries for charged particle motion [5], we do not start with a prescribed electromagnetic field. Rather, we find the conditions on $\mathbf{E}$ and $\mathbf{B}$ so that the system (1) 2) do admit a Lie point symmetry. With this strategy in mind we do not stick to any particular electromagnetic field but search for the most general form that may present the symmetry. Once the general forms are known they can eventually be specified in more detail to fit some particular application. In fact, electromagnetic fields written in terms of arbitrary functions are of fundamental importance for treating the Vlasov-

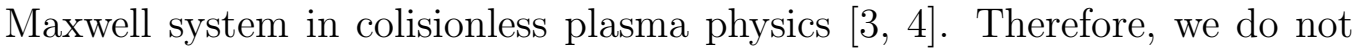
consider, in the continuation, any choice implying a too much particular field configuration, like that homogeneous in space. This strategy excludes some of the Lie point symmetries. Nevertheless, the approach gives the most general electromagnetic fields containing a number of arbitrary functions, that can be fixed later to fit particular applications like those of plasma physics.

As in the Noether point symmetry case [1], the Lie point symmetry approach also produces a system of linear, first order partial differential equations to be satisfied by the electromagnetic fields. We solve this system using a strategy similar to that used in reference [1]. That is, we find the canonical 
group coordinates for the various Lie symmetries and express the resulting system of partial differential equations in these coordinates. As a consequence, the system is transformed in a set of ordinary differential equations that can be solved easily. Our procedure shows how the problem of finding classes of electromagnetic fields compatible with Lie point symmetries is equivalent to the problem of finding canonical group variables for these same symmetries.

The paper is organized as follows. In section 2, we obtain the most general form of the Lie point symmetries associated with planar, non-relativistic charged particle motion. In the same section, we obtain the system of equations satisfied by the corresponding electromagnetic field. Section 3 is dedicated to the calculation of the canonical group variables for the Lie point symmetries. We find four classes of canonical coordinates. In section 4 , the basic system of partial differential equations satisfied by the electromagnetic field is solved for each of the four classes of canonical group variables. Section 5 is devoted to the conclusions.

\section{Lie point symmetries}

Let us consider infinitesimal point transformations,

$$
\begin{aligned}
\bar{x} & =x+\varepsilon \eta_{1}(x, y, t), \\
\bar{y} & =y+\varepsilon \eta_{2}(x, y, t), \\
\bar{t} & =t+\varepsilon \tau(x, y, t),
\end{aligned}
$$

where $\varepsilon$ is an infinitesimal parameter. For future convenience we denote the generator of the group of symmetries associated to (4 6) by

$$
G=\tau \frac{\partial}{\partial t}+\eta_{1} \frac{\partial}{\partial x}+\eta_{2} \frac{\partial}{\partial y}
$$

The generator $G$ appears frequently in what follows and is useful in the definition of canonical group coordinates, which plays a central role in the systematic determination of the electromagnetic fields associated the symmetries.

The condition for Lie symmetry [6, 7] reads, in this case

$$
G^{[2]}(\mathbf{N})_{\mathbf{N}=0}=0,
$$


where $\mathbf{N}=\left(N_{1}, N_{2}\right)$,

$$
\begin{aligned}
& N_{1}=\ddot{x}-E_{1}(x, y, t)-\dot{y} B(x, y, t), \\
& N_{2}=\ddot{y}-E_{2}(x, y, t)+\dot{x} B(x, y, t),
\end{aligned}
$$

$G^{[2]}$ being the generator of the twice extend group (for its complete expression see, for instance, references [6, 7]). The calculation of Lie point symmetries is a fairly known procedure and we only outline its main steps here. By inserting the equations of motion into the Lie symmetry criteria (7) we obtain a polynomial equation in the velocity components. For this polynomial form, condition (7) implies that the coefficients of all monomials of the form $\dot{x}^{m} \dot{y}^{n}$ must be identically zero. This yields a system of partial differential equations to be satisfied by $\tau, \eta_{1}$ and $\eta_{2}$. For instance, the terms cubic in velocity give a system with general solution

$$
\tau=\rho^{2}(t)+g_{1}(t) x+g_{2}(t) y,
$$

where $g_{1}, \ldots, g_{6}$ are arbitrary functions of the indicated arguments. Equation (10) wil be taken into account in the continuation.

The terms quadratic in the velocity yield

$$
\begin{aligned}
\eta_{1 x x}-2 \dot{g}_{1}+g_{2} B & =0, & & \eta_{1 x y}-\dot{g}_{2}-g_{1} B=0 \\
\eta_{1 y y}-g_{2} B & =0, & & \eta_{2 x x}+g_{1} B=0 \\
\eta_{2 x y}-\dot{g}_{1}+g_{2} B & =0, & & \eta_{2 y y}-2 \dot{g}_{2}-g_{1} B=0
\end{aligned}
$$

where we have used subscripts to denote partial derivatives. Direct inspection shows that the choice

$$
g_{1}=g_{2}=0
$$

keeps $B$ arbitrary and implies that $\eta_{1}$ and $\eta_{2}$ are linear functions of position,

$$
\begin{aligned}
& \eta_{1}=g_{3}(t) x+g_{4}(t) y+a_{1}(t), \\
& \eta_{2}=g_{5}(t) x+g_{6}(t) y+a_{2}(t),
\end{aligned}
$$

where $g_{3}, g_{4}, g_{5}, g_{6}, a_{1}$ and $a_{2}$ are functions of time only. It is now worth to stress that the choice (14) implies no restriction on the magnetic field, which remains completely arbitrary. Moreover, a detailed calculation involving equations (11-13) shows that the only way of keeping spatial dependence 
in the magnetic field is to set $g_{1}=g_{2}=0$. In fact, we are interested in classes of magnetic fields more general than simply those homogeneous in space. Hence, we adopt (14), with the corresponding solution (15-16) for $\eta_{1}$ and $\eta_{2}$. The important point here is that, up to this stage, we have preserved the complete arbitrariness of the magnetic field.

The terms linear in velocity yield

$$
\begin{aligned}
\left(g_{4}+g_{5}\right) B & =-2\left(\rho \ddot{\rho}+\dot{\rho}^{2}\right)+2 \dot{g}_{3}, \\
\left(g_{4}+g_{5}\right) B & =2\left(\rho \ddot{\rho}+\dot{\rho}^{2}\right)-2 \dot{g}_{6}, \\
G B & =\left(g_{3}-g_{6}-2 \rho \dot{\rho}\right) B+2 \dot{g}_{4}, \\
G B & =\left(g_{6}-g_{3}-2 \rho \dot{\rho}\right) B-2 \dot{g}_{5},
\end{aligned}
$$

where $G$ is the generator defined in (6).

An examination of equations (17 18$)$ shows that the only way of preserving space dependence in the magnetic field is to set

$$
g_{4}=-g_{5}=-\Omega(t)
$$

where $\Omega$ is a function of time only. Moreover, this implies, from (17 18),

$$
g_{3}=\rho \dot{\rho}+k_{1}, \quad g_{6}=\rho \dot{\rho}+k_{2},
$$

with, $k_{1}$ and $k_{2}$ numerical constants. Equation (22) and compatibility between equations (19 20) give

$$
\left(k_{1}-k_{2}\right) B=0
$$

For $B$ not identically zero, the conclusion is

$$
k_{1}=k_{2}=k
$$

where $k$ is a numerical constant. Equations (19 20) now furnishes

$$
G B=-2 \rho \dot{\rho} B-2 \dot{\Omega},
$$

where the generator of Lie symmetries may be split into four components

$$
G=G_{Q}+G_{R}+G_{T}+G_{S}
$$


In equation (26),

$$
G_{Q}=\rho^{2}(t) \frac{\partial}{\partial t}+\rho \dot{\rho}\left(x \frac{\partial}{\partial x}+y \frac{\partial}{\partial y}\right),
$$

is the generator of quasi-invariance transformations [ [8],

$$
G_{R}=\Omega(t)\left(x \frac{\partial}{\partial y}-y \frac{\partial}{\partial x}\right)
$$

generates time-dependent rotations,

$$
G_{T}=a_{1}(t) \frac{\partial}{\partial x}+a_{2}(t) \frac{\partial}{\partial y}
$$

generates time-dependent spatial translations and

$$
G_{S}=k\left(x \frac{\partial}{\partial x}+y \frac{\partial}{\partial y}\right)
$$

is the generator of dilatations (or, more precisely, of contractions if $k<$ $0)$. Comparison with the generator of Noether point symmetries for twodimensional non-relativistic charged-particle motion [1] shows that the Lie point symmetry generator has an additional term, depending on the parameter $k$. We also observe that this form is essentially new, and cannot be expressed in terms of the generators $G_{Q}, G_{R}$ and $G_{T}$.

The terms independent of velocity in the Lie invariance condition have also to be taken into account. They produce the equations for the electric field,

$$
\begin{aligned}
G E_{1}=(-3 \rho \dot{\rho} & +k) E_{1}-\Omega E_{2}-\left(\left(\rho \ddot{\rho}+\dot{\rho}^{2}\right) y+\dot{\Omega} x+\dot{a}_{2}\right) B+ \\
& +(\rho \ddot{\rho}+3 \dot{\rho} \ddot{\rho}) x-\ddot{\Omega} y+\ddot{a}_{1}, \\
G E_{2}=(-3 \rho \dot{\rho} & +k) E_{2}+\Omega E_{1}+\left(\left(\rho \ddot{\rho}+\dot{\rho}^{2}\right) x-\dot{\Omega} y+\dot{a}_{1}\right) B+ \\
& +(\rho \ddot{\rho}+3 \dot{\rho} \ddot{\rho}) y+\ddot{\Omega} x+\ddot{a}_{2} .
\end{aligned}
$$

Let us summarize the results obtained so far. Implicitly, in our treatment, we excluded the excessively restricted class of spatially homogeneous magnetic fields depending on time only. This approach yields the system 
equations (25), (31-32), which must be satisfied by the electromagnetic fields associated to Lie point symmetries of the planar charged particle motion. The Lie symmetry generator in equations (25), (31 32), which constitute a system of linear, first order, coupled partial differential equations for $E_{1}, E_{2}$ and $B$, is given by (26). In comparison with the treatment of two-dimensional non-relativistic charged particle motion with Noether point symmetries, we find that Lie point symmetries have an extra component associated to scale transformation. This extra contribution modifies both the generator of symmetries and the equations satisfied by the electromagnetic fields [1].

In the remaining of this paper, we are essentially concerned with finding all the solutions of the system of partial differential equations (25), (31 32). These solutions yield the most general electromagnetic field under which the planar motion of charged particles present Lie point symmetry. An useful remark is that $B$ satisfies an equation decoupled from the equations for $E_{1}$ and $E_{2}$, whereas the equations for the electric field do depend on $B$. Thus, we must first solve (25) for $B$ and only afterwards treat (31 32) for the electric field.

Finally, if these solutions should constitute true electromagnetic fields, the additional requirement of Faraday's law

$$
E_{2 x}-E_{1 y}+B_{t}=0,
$$

must be imposed, to comply with Maxwell's equations.

To treat the system (25), (31 [32) and to find its complete solution, we shall use canonical group coordinates. These variables are introduced in the section that follows.

\section{Canonical group coordinates}

Canonical group coordinates [6, [7] are defined by imposing that the symmetry transformation behaves merely like time translation. Denoting new coordinates by $(\bar{x}, \bar{y}, \bar{t})$, this means that, in canonical group coordinates,

$$
G=\frac{\partial}{\partial \bar{t}}
$$

where $\bar{t}$ is the new time parameter. This implies that canonical group coordinates satisfy the equations

$$
G \bar{x}=0, \quad G \bar{y}=0, \quad G \bar{t}=1 .
$$


This set of uncoupled linear partial differential equations, can be solved, for the generator (26), in closed form by the method of characteristics. We find four classes of solutions, listed below.

\subsection{The case $\rho \neq 0$}

When $\rho \neq 0$, it is convenient to write

$$
\begin{aligned}
& a_{1}=\rho^{2} \dot{\alpha}_{1}-(\rho \dot{\rho}+k) \alpha_{1}, \\
& a_{2}=\rho^{2} \dot{\alpha}_{2}-(\rho \dot{\rho}+k) \alpha_{2}
\end{aligned}
$$

for suitable functions $\alpha_{1}(t)$ and $\alpha_{2}(t)$, which are therefore defined in terms of $a_{1}$ and $a_{2}$.

In terms of (36 37), we have the following canonical group coordinates,

$$
\begin{aligned}
& \bar{t}=\int^{t} d \mu / \rho^{2}(\mu), \\
& \bar{x}=\frac{e^{-k \bar{t}}}{\rho}\left(\left(x-\alpha_{1}\right) \cos T+\left(y-\alpha_{2}\right) \sin T\right)+\delta_{1}, \\
& \bar{y}=\frac{e^{-k \bar{t}}}{\rho}\left(-\left(x-\alpha_{1}\right) \sin T+\left(y-\alpha_{2}\right) \cos T\right)+\delta_{2},
\end{aligned}
$$

where new functions $T=T(t), \delta_{1}=\delta_{1}(t)$ and $\delta_{2}=\delta_{2}(t)$ were defined according to

$$
\begin{aligned}
T(t) & =\int^{t} d \mu \Omega(\mu) / \rho^{2}(\mu) \\
\delta_{1}(t) & =-\int^{t} d \mu \frac{\Omega(\mu)}{\rho^{3}(\mu)} e^{-k \bar{t}(\lambda)}\left(\alpha_{1}(\mu) \sin T(\mu)-\alpha_{2}(\mu) \cos T(\mu)\right) \\
\delta_{2}(t) & =-\int^{t} d \mu \frac{\Omega(\mu)}{\rho^{3}(\mu)} e^{-k \bar{t}(\lambda)}\left(\alpha_{1}(\mu) \cos T(\mu)+\alpha_{2}(\mu) \sin T(\mu)\right) .
\end{aligned}
$$

Notice that the requirement $\rho \neq 0$ is essential, for otherwise the canonical group variables $(38$ 40) would not be well defined.

For $k=\Omega=\alpha_{1}=\alpha_{2}=0$, the transformation (38 40) is known as the quasi-invariance transformation [8]. In the general case, however, the transformations includes also dilatation, time-dependent rotation and time 
dependent translation. In comparison with the Noether point symmetry approach, the present set of canonical group variables are in direct correspondence with case 3.1 of reference [1]. In fact, when $k=0$, formulae (38 40) become formulae (42-44) of [1].

\subsection{The case $\rho=k=0$ and $\Omega \neq 0$}

In this case, we have Noether point symmetry [1]. The canonical group variables are

$$
\begin{aligned}
\bar{t} & =\frac{1}{\Omega} \tan ^{-1}\left(\frac{y-\beta_{2}}{x-\beta_{1}}\right) \\
\bar{x} & =\left(\left(x-\beta_{1}\right)^{2}+\left(y-\beta_{2}\right)^{2}\right)^{1 / 2} \\
\bar{y} & =t
\end{aligned}
$$

with

$$
\beta_{1}=\beta_{1}(t)=-a_{2} / \Omega \quad, \quad \beta_{2}=\beta_{2}(t)=a_{1} / \Omega \quad .
$$

The variables $\bar{x}$ and $\bar{t}$ are translated polar coordinates, the new time parameter playing the role of an azimuthal angle and $\bar{x}$ the role of a radial coordinate.

\subsection{The case $\rho=k=\Omega=0$ and $a_{2} \neq 0$}

Again, we have Noether point symmetry with canonical group variables

$$
\begin{aligned}
\bar{t} & =y / a_{2}, \\
\bar{x} & =x-a_{1} y / a_{2}, \\
\bar{y} & =t .
\end{aligned}
$$

We finally mention that the case $\rho=0, \Omega=0$ and $a_{1} \neq 0$ is strictly analogous to this last case and deserves no special consideration.

\subsection{The case $\rho=0, k \neq 0$.}

In this case the canonical coordinates are

$$
\bar{t}=\frac{1}{2 k} \log \left(\left(x-\gamma_{1}\right)^{2}+\left(y-\gamma_{2}\right)^{2}\right),
$$




$$
\begin{aligned}
& \bar{x}=\arctan \left(\frac{y-\gamma_{2}}{x-\gamma_{1}}\right)-\Omega \bar{t}, \\
& \bar{y}=t,
\end{aligned}
$$

where

$$
\gamma_{1}=-\frac{k a_{1}+\Omega a_{2}}{k^{2}+\Omega^{2}}, \quad \gamma_{2}=\frac{\Omega a_{1}-k a_{2}}{k^{2}+\Omega^{2}} .
$$

The symmetry transformation represents a dilatation, plus a time-dependent rotation and a time-dependent translation.

\section{Electromagnetic fields}

We now tackle equations (25), (31 32) for the electromagnetic fields in each of the four possible symmetry transformations expressed in canonical group variables.

\subsection{The case $\rho \neq 0$}

Equation (25), which involves only the magnetic field acquires, in canonical group coordinates, the form

$$
B_{\bar{t}}=-\frac{2 \rho^{\prime}}{\rho} B-\frac{2 \Omega^{\prime}}{\rho^{2}},
$$

where prime denotes total differentiation with respect to $\bar{t}$. The general solution for (55) is

$$
B=-\frac{2 \Omega}{\rho^{2}}+\frac{1}{\rho^{2}} \bar{B}(\bar{x}, \bar{y}),
$$

where $\bar{B}(\bar{x}, \bar{y})$ is an arbitrary function of the indicated arguments. Notice that the resulting magnetic field is not necessarily homogeneous, since it can depend on the spatial coordinates through $\bar{x}$ and $\bar{y}$. This is a significant improvement on earlier results [9]. Formally, $B$ is identical to the magnetic field of case 4.1 in reference [1] on Noether point symmetries. Notice, however, the different form of canonical group variables.

To find the corresponding electric field, we must solve the system (31 32), taking the solution (56) into account. In this case, it is useful to introduce 
the quantities $\Sigma_{1}$ and $\Sigma_{2}$ defined by

$$
\begin{aligned}
& \Sigma_{1}=\rho^{3} e^{-k \bar{t}}\left(E_{1} \cos T+E_{2} \sin T\right), \\
& \Sigma_{2}=\rho^{3} e^{-k \bar{t}}\left(-E_{1} \sin T+E_{2} \cos T\right),
\end{aligned}
$$

representing a rotation plus a rescaling of the electric field which, in this case, can be viewed as of a circularly polarized wave with time-dependent amplitude. In the new variables, the system (31-32) decouples and can be cast into the form

$$
\frac{\partial \Sigma_{1}}{\partial \bar{t}}=\frac{\partial \psi_{1}}{\partial \bar{t}} \quad, \quad \frac{\partial \Sigma_{2}}{\partial \bar{t}}=\frac{\partial \psi_{2}}{\partial \bar{t}}
$$

where

$$
\begin{aligned}
\psi_{1} & =\left(-\frac{\rho^{\prime}}{\rho}\left(\bar{y}-\delta_{2}\right)+\delta_{2}^{\prime}+k \delta_{2}-\Omega\left(\bar{x}-\delta_{1}\right)+\frac{e^{-k \bar{t}}}{\rho}\left(\alpha_{1}^{\prime} \sin T-\alpha_{2}^{\prime} \cos T\right)\right) \bar{B}(\bar{x}, \bar{y})+ \\
& +\left(\frac{\rho^{\prime \prime}}{\rho}-2 \frac{\rho^{\prime 2}}{\rho^{2}}+\Omega^{2}\right)\left(\bar{x}-\delta_{1}\right)-\left(\Omega^{\prime}-2 \frac{\rho^{\prime}}{\rho} \Omega\right)\left(\bar{y}-\delta_{2}\right)+ \\
& +\frac{e^{-k \bar{t}}}{\rho}\left(\Omega^{\prime} \alpha_{1}-\Omega\left(\alpha_{1}^{\prime}+\frac{\rho^{\prime}}{\rho} \alpha_{1}\right)+\alpha_{2}^{\prime \prime}-2 \frac{\rho^{\prime}}{\rho} \alpha_{2}^{\prime}+\Omega^{2} \alpha_{2}\right) \sin T+ \\
& +\frac{e^{-k \bar{t}}}{\rho}\left(-\Omega^{\prime} \alpha_{2}+\Omega\left(\alpha_{2}^{\prime}+\frac{\rho^{\prime}}{\rho} \alpha_{2}\right)+\alpha_{1}^{\prime \prime}-2 \frac{\rho^{\prime}}{\rho} \alpha_{1}^{\prime}+\Omega^{2} \alpha_{1}\right) \cos T \\
& -k\left(\delta_{1}^{\prime}+k \delta_{1}\right), \\
\psi_{2} & \left(+\frac{\rho^{\prime}}{\rho}\left(\bar{x}-\delta_{1}\right)-\delta_{1}^{\prime}-k \delta_{1}-\Omega\left(\bar{y}-\delta_{2}\right)+\frac{e^{-k \bar{t}}}{\rho}\left(\alpha_{1}^{\prime} \cos T+\alpha_{2}^{\prime} \sin T\right)\right) \bar{B}(\bar{x}, \bar{y})+ \\
& +\left(\frac{\rho^{\prime \prime}}{\rho}-2 \frac{\rho^{\prime 2}}{\rho^{2}}+\Omega^{2}\right)\left(\bar{y}-\delta_{2}\right)+\left(\Omega^{\prime}-2 \frac{\rho^{\prime}}{\rho} \Omega\right)\left(\bar{x}-\delta_{1}\right) \\
& -\frac{e^{-k \bar{t}}}{\rho}\left(-\Omega^{\prime} \alpha_{2}+\Omega\left(\alpha_{2}^{\prime}+\frac{\rho^{\prime}}{\rho} \alpha_{2}\right)+\alpha_{1}^{\prime \prime}-2 \frac{\rho^{\prime}}{\rho} \alpha_{1}^{\prime}+\Omega^{2} \alpha_{1}\right) \sin T+ \\
& +\frac{e^{-k \bar{t}}}{\rho}\left(+\Omega^{\prime} \alpha_{1}-\Omega\left(\alpha_{1}^{\prime}+\frac{\rho^{\prime}}{\rho} \alpha_{1}\right)+\alpha_{2}^{\prime \prime}-2 \frac{\rho^{\prime}}{\rho} \alpha_{2}^{\prime}+\Omega^{2} \alpha_{2}\right) \cos T \\
& -k\left(\delta_{2}^{\prime}+k \delta_{2}\right) .
\end{aligned}
$$

The general solution for (59) is

$$
\Sigma_{1}=\psi_{1}+\bar{E}_{1}(\bar{x}, \bar{y}) \quad, \quad \Sigma_{2}=\psi_{2}+\bar{E}_{2}(\bar{x}, \bar{y}),
$$


where, as indicated, $\bar{E}_{1}$ and $\bar{E}_{2}$ have no dependence on $\bar{t}$.

We are interested in the electric field, in the original variables. To obtain the field in this coordinates we use the inverse of the transformation (57 58),

$$
\begin{aligned}
& E_{1}=\frac{e^{k \bar{t}}}{\rho^{3}}\left(\Sigma_{1} \cos T-\Sigma_{2} \sin T\right), \\
& E_{2}=\frac{e^{k \bar{t}}}{\rho^{3}}\left(\Sigma_{1} \sin T+\Sigma_{2} \cos T\right) .
\end{aligned}
$$

Substituting equations (63 64) into (62) and transforming back to the original variables $(x, y, t)$, yields the electric field components

$$
\begin{aligned}
& E_{1}=\ddot{\alpha}_{1}+\frac{\ddot{\rho}}{\rho}\left(x-\alpha_{1}\right)+\frac{\Omega^{2} x}{\rho^{4}}-(\rho \dot{\Omega}-2 \dot{\rho} \Omega) \frac{y}{\rho^{3}}+\frac{\Omega}{\rho^{3}}\left(\rho \dot{\alpha}_{2}-\dot{\rho} \alpha_{2}\right)+ \\
& +\frac{k^{2} e^{k \bar{t}}}{\rho^{3}}\left(\delta_{2} \sin T-\delta_{1} \cos T\right)-\frac{k \Omega \alpha_{2}}{\rho^{4}}+ \\
& +\frac{e^{k \bar{t}}}{\rho^{3}}\left(\bar{E}_{1}(\bar{x}, \bar{y}) \cos T-\bar{E}_{2}(\bar{x}, \bar{y}) \sin T\right) \\
& -\frac{1}{\rho^{4}}\left(\rho \dot{\rho}\left(y-\alpha_{2}\right)+\rho^{2} \dot{\alpha}_{2}+\Omega x-k \rho e^{k \bar{t}}\left(\delta_{2} \cos T+\delta_{1} \sin T\right)\right) \bar{B}(\bar{x}, \bar{y}), \\
& E_{2}=\ddot{\alpha}_{2}+\frac{\ddot{\rho}}{\rho}\left(y-\alpha_{2}\right)+\frac{\Omega^{2} y}{\rho^{4}}+(\rho \dot{\Omega}-2 \dot{\rho} \Omega) \frac{x}{\rho^{3}}-\frac{\Omega}{\rho^{3}}\left(\rho \dot{\alpha}_{1}-\dot{\rho} \alpha_{1}\right) \\
& -\frac{k^{2} e^{k \bar{t}}}{\rho^{3}}\left(\delta_{2} \cos T+\delta_{1} \sin T\right)+\frac{k \Omega \alpha_{1}}{\rho^{4}}+ \\
& +\frac{e^{k \bar{t}}}{\rho^{3}}\left(\bar{E}_{2}(\bar{x}, \bar{y}) \cos T+\bar{E}_{1}(\bar{x}, \bar{y}) \sin T\right)+ \\
& +\frac{1}{\rho^{4}}\left(\rho \dot{\rho}\left(x-\alpha_{1}\right)+\rho^{2} \dot{\alpha}_{1}-\Omega y-k \rho e^{k \bar{t}}\left(\delta_{1} \cos T-\delta_{2} \sin T\right)\right) \bar{B}(\bar{x}, \bar{y}) .
\end{aligned}
$$

It still remains to take into consideration Faraday's law, which, in our case, is equivalent to eq. (33). After a detailed calculation using the magnetic field (56) and the electric field (65 66), we find that Faraday's law imposes

$$
\bar{E}_{2 \bar{x}}-\bar{E}_{1 \bar{y}}=k\left(\bar{x} \bar{B}_{\bar{x}}+\bar{y} \bar{B}_{\bar{y}}\right) .
$$


For $k=0$ (the Noether point symmetry subcase), equation (67) has the general solution

$$
\bar{E}_{1}=-\frac{\partial}{\partial \bar{x}} \bar{V}(\bar{x}, \bar{y}) \quad, \quad \bar{E}_{2}=-\frac{\partial}{\partial \bar{y}} \bar{V}(\bar{x}, \bar{y}) \quad,
$$

where $\bar{V}(\bar{x}, \bar{y})$ is an arbitrary function of the indicated argument. For $k \neq 0$ equation (67) is a different constraint to be imposed on the electromagnetic field.

In conclusion, we have obtained a very general class of electromagnetic fields yielding Lie point symmetries. The magnetic field is given by eq. (56) and the electric field by eqs. (65-66), together with condition (67). The electromagnetic field involves several arbitrary functions, namely $\rho(t), \alpha_{1}(t)$, $\alpha_{2}(t), \Omega(t), \bar{B}(\bar{x}, \bar{y})$ and $\bar{E}_{1}(\bar{x}, \bar{y})$ or $\bar{E}_{2}(\bar{x}, \bar{y})$, where $\bar{x}$ and $\bar{y}$ are defined by eqs. (39 40). For instance, for given $\bar{B}$ and $\bar{E}_{1}$ the constraint (67) defines $\bar{E}_{2}$ up to the addition of an arbitrary function of $\bar{y}$.

To conclude this subsection, let us write the equations of motion in transformed coordinates,

$$
\begin{aligned}
\bar{x}^{\prime \prime}+2 k \bar{x}^{\prime}+k^{2} \bar{x} & =\bar{E}_{1}(\bar{x}, \bar{y})+\left(\bar{y}^{\prime}+k \bar{y}\right) \bar{B}(\bar{x}, \bar{y}), \\
\bar{y}^{\prime \prime}+2 k \bar{y}^{\prime}+k^{2} \bar{y} & =\bar{E}_{2}(\bar{x}, \bar{y})-\left(\bar{x}^{\prime}+k \bar{x}\right) \bar{B}(\bar{x}, \bar{y}) .
\end{aligned}
$$

As they stand, these equations are not integrable in the general case.

\subsection{The case $\rho=0, k=0$ and $\Omega \neq 0$}

In this case we have Noether point symmetry. Hence, we simply quote the main results from reference [1]. The electromagnetic field is given by

$$
\begin{aligned}
B & =\bar{B}(\bar{x}, \bar{y}), \\
E_{1} & =\ddot{\beta}_{1}-\dot{\beta}_{2} \bar{B}(\bar{x}, \bar{y})+ \\
& +\left(x-\beta_{1}\right) \bar{E}_{1}(\bar{x}, \bar{y})-\left(y-\beta_{2}\right) \bar{E}_{2}(\bar{x}, \bar{y}), \\
E_{2} & =\ddot{\beta}_{2}+\dot{\beta}_{1} \bar{B}(\bar{x}, \bar{y})+ \\
& +\left(x-\beta_{1}\right) \bar{E}_{2}(\bar{x}, \bar{y})+\left(y-\beta_{2}\right) \bar{E}_{1}(\bar{x}, \bar{y}),
\end{aligned}
$$

where $\bar{B}, \bar{E}_{1}$ and $\bar{E}_{2}$ are arbitrary functions of $\bar{x}, \bar{y}$ given in equations (45 46).

Faraday's law requires

$$
\bar{x} \bar{E}_{2 \bar{x}}+2 \bar{E}_{2}=-\bar{B}_{\bar{y}}
$$


whose solution is

$$
\bar{E}_{2}=\frac{1}{\bar{x}^{2}} \frac{\partial \psi}{\partial \bar{y}} \quad, \quad \bar{B}=-\frac{1}{\bar{x}} \frac{\partial \psi}{\partial \bar{x}} \quad
$$

for arbitrary $\psi=\psi(\bar{x}, \bar{y})$.

In conclusion, the electromagnetic field is given by eqs. (71 73$)$, with the constraint (75). There remains four arbitrary functions, namely $E_{1}(\bar{x}, \bar{y})$, $\psi(\bar{x}, \bar{y}), \beta_{1}(t)$ and $\beta_{2}(t)$, with $\bar{x}, \bar{y}$ defined in equations (45 46). We also observe that in the present case $\Omega(t)$ has to be chosen constant in order to produce physically meaningful electromagnetic field (for details, see [1]). Without loss of generality, we take $\Omega=1$.

\subsection{The case $\rho=0, k=0, \Omega=0$ and $a_{2} \neq 0$}

Again we have Noether point symmetry. The eletromagnetic fields, from ref. [闻], are

$$
\begin{aligned}
B & =\bar{B}(\bar{x}, \bar{y}), \\
E_{1} & =\frac{\ddot{a}_{1} y}{a_{2}}-\frac{\dot{a}_{2} y}{a_{2}} \bar{B}(\bar{x}, \bar{y})+\bar{E}_{1}(\bar{x}, \bar{y}), \\
E_{2} & =\frac{\ddot{a}_{2} y}{a_{2}}+\frac{\dot{a}_{1} y}{a_{2}} \bar{B}(\bar{x}, \bar{y})+\bar{E}_{2}(\bar{x}, \bar{y}),
\end{aligned}
$$

where $\bar{B}, \bar{E}_{1}$ and $\bar{E}_{2}$ are arbitrary functions and $\bar{x}, \bar{y}$ are defined in equations (49 50).

After solving the differential equations arising from Noether's symmetry condition, we must verify the constraint imposed by Faraday's law, which, in this case, implies

$$
\begin{aligned}
\bar{B} & =\psi_{\bar{x}}, \\
\bar{E}_{1} & =-\bar{V}_{\bar{x}}, \\
\bar{E}_{2} & =\frac{\ddot{a}_{1}}{a_{2}} \bar{x}-\frac{\dot{a}_{2}}{a_{2}} \psi-\psi_{\bar{y}}+\frac{a_{1}}{a_{2}} \bar{V}_{\bar{x}} .
\end{aligned}
$$

Here, $\psi=\psi(\bar{x}, \bar{y})$ and $\bar{V}=\bar{V}(\bar{x}, \bar{y})$ are arbitrary functions.

This completely determines this class of solutions for the electromagnetic field. $B$ is given by eq. (76) and $E_{1}$ and $E_{2}$ are given by eqs. (77 78). The functions $\bar{B}, \bar{E}_{1}$ and $\bar{E}_{2}$, appearing in the solution, are given by eqs. 81), in terms of the arbitrary functions $\psi(\bar{x}, \bar{y})$ and $\bar{V}(\bar{x}, \bar{y})$ with $\bar{x}, \bar{y}$ given 
by (49 50). The arbitrary functions $a_{1}(t)$ and $a_{2}(t)$ are also present in the electromagnetic field, so that four arbitrary functions participate in the final solution.

\subsection{The case $\rho=0$ and $k \neq 0$}

In this case the equation for the magnetic field is

$$
B_{\bar{t}}=-2 \dot{\Omega}(\bar{y}),
$$

with solution

$$
B=-2 \dot{\Omega}(\bar{y}) \bar{t}+\bar{B}(\bar{x}, \bar{y}) .
$$

Inserting this magnetic field in the equations for the electric field, yields

$$
\begin{aligned}
& E_{1 \bar{t}}=k E_{1}-\Omega E_{2}+\left(\dot{\Omega} x+\dot{a}_{2}\right)(2 \dot{\Omega} \bar{t}-\bar{B})-\ddot{\Omega} y+\ddot{a}_{1}, \\
& E_{2 \bar{t}}=k E_{2}+\Omega E_{1}+\left(\dot{\Omega} y-\dot{a}_{1}\right)(2 \dot{\Omega} \bar{t}-\bar{B})+\ddot{\Omega} x+\ddot{a}_{2} .
\end{aligned}
$$

This system may be handled with the more convenient variables

$$
\begin{aligned}
& \Sigma_{1}=e^{-k \bar{t}}\left(E_{1} \cos \Omega \bar{t}+E_{2} \sin \Omega \bar{t}\right), \\
& \Sigma_{2}=e^{-k \bar{t}}\left(-E_{1} \sin \Omega \bar{t}+E_{2} \cos \Omega \bar{t}\right) .
\end{aligned}
$$

Using these new variables, we have the transformed equations

$$
\frac{\partial \Sigma_{1}}{\partial \bar{t}}=\frac{\partial \psi_{1}}{\partial \bar{t}} \quad, \quad \frac{\partial \Sigma_{2}}{\partial \bar{t}}=\frac{\partial \psi_{2}}{\partial \bar{t}} \quad,
$$

where

$$
\begin{aligned}
\psi_{1} & =\left(\dot{\Omega}^{2} \bar{t}^{2}-\dot{\Omega} \bar{B} \bar{t}\right) \cos \bar{x}-\ddot{\Omega} \bar{t} \sin \bar{x}+ \\
& +\left(2 \dot{\Omega} \dot{\gamma}_{2} \bar{t}+\ddot{\gamma}_{1}-\dot{\gamma}_{2} \bar{B}\right) e^{-k \bar{t}} \cos \Omega \bar{t}+ \\
& +\left(-2 \dot{\Omega} \dot{\gamma}_{1} \bar{t}+\ddot{\gamma}_{2}+\dot{\gamma}_{1} \bar{B}\right) e^{-k \bar{t}} \sin \Omega \bar{t}, \\
\psi_{2} & =\left(\dot{\Omega}^{2} \bar{t}^{2}-\dot{\Omega} \bar{B} \bar{t}\right) \sin \bar{x}+\ddot{\Omega} \bar{t} \cos \bar{x}+ \\
& +\left(-2 \dot{\Omega} \dot{\gamma}_{1} \bar{t}+\ddot{\gamma}_{2}+\dot{\gamma}_{1} \bar{B}\right) e^{-k \bar{t}} \cos \Omega \bar{t} \\
& -\left(2 \dot{\Omega} \dot{\gamma}_{2} \bar{t}+\ddot{\gamma}_{1}-\dot{\gamma}_{2} \bar{B}\right) e^{-k \bar{t}} \sin \Omega \bar{t} .
\end{aligned}
$$


The solutions to (88) are

$$
\Sigma_{1}=\psi_{1}+\bar{E}_{1}(\bar{x}, \bar{y}) \quad, \quad \Sigma_{2}=\psi_{2}+\bar{E}_{2}(\bar{x}, \bar{y}),
$$

The inverse transformation for 86 87) is

$$
\begin{aligned}
& E_{1}=e^{k \bar{t}}\left(\Sigma_{1} \cos \Omega \bar{t}-\Sigma_{2} \sin \Omega \bar{t}\right), \\
& E_{2}=e^{k \bar{t}}\left(\Sigma_{1} \sin \Omega \bar{t}+\Sigma_{2} \cos \Omega \bar{t}\right) .
\end{aligned}
$$

Back in the original coordinates, the resulting electric field becomes

$$
\begin{aligned}
E_{1} & =\ddot{\gamma}_{1}+2 \dot{\Omega} \dot{\gamma}_{2} \bar{t}-\dot{\gamma}_{2} \bar{B}+\dot{\Omega} \bar{t}(\dot{\Omega} \bar{t}-\bar{B})\left(x-\gamma_{1}\right) \\
& -\ddot{\Omega} \bar{t}\left(y-\gamma_{2}\right)+e^{k \bar{t}}\left(\bar{E}_{1} \cos \Omega \bar{t}-\bar{E}_{2} \sin \Omega \bar{t}\right), \\
E_{2} & =\ddot{\gamma}_{2}-2 \dot{\Omega} \dot{\gamma}_{1} \bar{t}+\dot{\gamma}_{1} \bar{B}+\dot{\Omega} \bar{t}(\dot{\Omega} \bar{t}-\bar{B})\left(y-\gamma_{2}\right) \\
& +\ddot{\Omega} \bar{t}\left(x-\gamma_{1}\right)+e^{k \bar{t}}\left(\bar{E}_{1} \sin \Omega \bar{t}+\bar{E}_{2} \cos \Omega \bar{t}\right) .
\end{aligned}
$$

Here it is more convinient to use a hybrid notation with transformed time $\bar{t}$ in order to obtain simpler expressions. We should also stress the generality of the resulting electromagnetic field, which possesses six arbitrary functions, namely $\gamma_{1}, \gamma_{2}, \Omega, \bar{B}, \bar{E}_{1}$ and $\bar{E}_{2}$.

To finalize, the constraint arising from Faraday's law becomes

$$
\begin{aligned}
k \frac{\partial \bar{B}}{\partial \bar{y}} & =-\ddot{\Omega}(\bar{y})+(k \sin \bar{x}-\Omega(\bar{y}) \cos \bar{x}) \bar{E}_{1}-(k \cos \bar{x}+\Omega(\bar{y}) \sin \bar{x}) \bar{E}_{2} \\
& +\quad(k \cos \bar{x}-\Omega(\bar{y}) \sin \bar{x}) \frac{\partial \bar{E}_{1}}{\partial \bar{x}}+(k \sin \bar{x}+\Omega(\bar{y}) \cos \bar{x}) \frac{\partial \bar{E}_{2}}{\partial \bar{x}}
\end{aligned}
$$

This condition must be satisfied by the arbitrary functions appearing in the solution. For instance, after specifying $\Omega, \bar{E}_{1}$ and $\bar{E}_{2}$, we can consider (96) as an equation determining $\bar{B}$ up to the addition of an arbitrary function of $\bar{x}$.

\section{Conclusion}

We have found all classes of electromagnetic fields for which planar nonrelativistic charged particle motion is compatible with Lie point symmetries. 
Our procedure is based on the resolution of the basic system of linear firstorder partial differential equations (25), (31 32 satisfied by the electromagnetic field, using canonical group variables. As shown in section 2, there exist four types of canonical group variables, yielding four classes of electromagnetic fields compatible with Lie point symmetry. In comparison with the Noether point symmetry analysis [1], an additional dilatation invariance term appears in the generator of Lie point symmetries. This dilatation invariance is associated with an extra category of electromagnetic fields compatible with Lie point symmetries. The electromagnetic fields of subsections 4.2 and 4.3 just fit into the Noether point symmetry case. The electromagnetic field of section 4.1 can be viewed as a natural extension of the Noether point symmetry case treated in subsection 4.1 of reference [1]. The class shown in section 4.4 of the present work, however, is essentially new. Its origin can be traced back to the additional dilatation invariance which is not possible in the Noether's theorem framework.

In our treatment, we do not include some symmetries corresponding to excessively particular classes of electromagnetic fields homogeneous in space. In this way, we concentrate on classes of electromagnetic fields depending on arbitrary functions of certain similarity variables involving space coordinates. These classes may be useful, for example, in the search for new exact or approximate solutions for the Vlasov-Maxwell system in colisionless plasma physics. Also, as pointed out in the introduction, symmetry may help reduc-

ing the number of relevant coordinates of the problem and this may represent a considerable reduction in the cost of its numerical treatment.

\section{Acknowledgement}

This work has been partially supported by Fundação de Amparo a Pesquisa do Estado do Rio Grande do Sul (FAPERGS).

\section{References}

[1] F. Haas and J. Goedert, Noether symmetries for two-dimensional charged particle motion, accepted for publication on J. Phys. A: Math. Gen. (1999).

[2] W. Sarlet and F. Cantrijn, SIAM Rev. 23 (1981) 467. 
[3] H. R. Lewis and K. R. Symon, Phys. Fluids 27 (1984) 192.

[4] B. Abraham-Schrauner, Phys. Fluids 27 (1984) 197.

[5] O. M. Ritter, Simetrias de Lie para a Partícula Carregada sob Campos Eletromagnéticos Gerais, MsC. Thesis, UFRJ (1991).

[6] G. W. Bluman and J. D. Cole, Similarity Methods for Differential Equations (Springer, New York, 1974).

[7] P. G. L. Leach, Differential Equations, Symmetries and Integrability, Lecture Notes, Université d'Orléans (1996).

[8] A. Munier, J. R. Burgan, M. Feix and E. Fijalkow, J. Math. Phys. 22 (1981) 1219.

[9] See, e. g., S. Bouquet, L. Cairó and M. R. Feix, J. Plasma Phys. 34 (1985) 127. 